
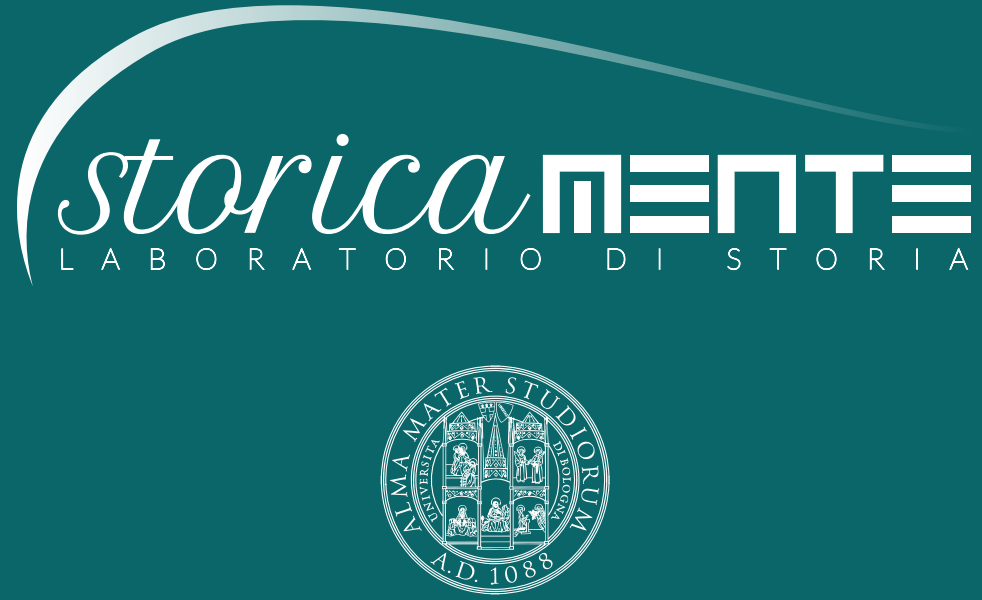

ALMA MATER STUDIORUM

Università di Bologna

Dipartimento di Storia Culture Civiltà

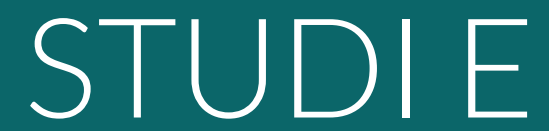

RICERCHE 


\section{STORICAMENTE.ORG}

\section{Laboratorio di Storia}

\section{Andrea Griffante}

La Prima guerra mondiale e l'uso pubblico della storia in Lituania: i nuovi Cavalieri teutonici

Numero 10 - 2014

ISSN: $1825-411 \mathrm{X}$

Art. 18

pp. 1-25

DOI: $10.12977 /$ stor569

Editore: BraDypUS

Data di pubblicazione: 09/10/2014

Sezione: Studi e Ricerche 


\title{
La Prima guerra mondiale e l'uso pubblico della storia in Lituania: i nuovi Cavalieri teutonici
}

\author{
ANDREA GRIFFANTE \\ Vytautas Magnus University, Department of Political \\ Sciences and Diplomacy, Gedimino g. 44, LT-44240 \\ Kaunas, Lithuania
}

Allo scoppio della Prima guerra mondiale, la mobilitazione delle masse rappresentò un compito a cui, seppur per ragioni differenti, né le forze imperiali, néle élite intellettuali nazionali dell'Impero zarista poterono sottrarsi. Simboli ed elementi delle diverse mitologie nazionali furono impiegate come strumenti di mobilitazione culturale. A questo fine, gli intellettuali lituani impiegarano ampiamente la memoria della battaglia di Grünwald (1410) e dei "Cavalieri teutonici". Allorché l'occupazione tedesca ebbe inizio, la categoria di "brutalità teutonica" iniziò a essere utilizzata per dar senso a un conflitto di dimensioni mai viste prima.

At the outburst of the First World War, the mobilization of masses represented, even if for different reasons, an unavoidable goal for both the Imperial powers and national intellectual groups operating in the Tsarist Empire. Symbols and elements of national mythology were often used as cultural mobilization instruments. Lithuanian intellectuals based their mobilization work mainly on the memory of the Battle of Grünwald (1410). When the German occupation began, the Lithuanians used the category of "Teutonic bestiality" to make sense of their experience.

Il presente lavoro è stato realizzato grazie al finanziamento del Consiglio Lituano delle Scienze (contratto n. LIT-6-8). La sua realizzazione è avvenuta in collaborazione con l'Istituto Lituano di Storia. 
Quando, nell'estate del 1914, l'Impero zarista iniziò a mobilitare le proprie forze dopo la dichiarazione di guerra della Germania, l'avvicinarsi del conflitto fu accolto dalla società lituana dell'Impero' con un misto di sconcerto e speranza. Per la gran parte della popolazione la guerra rappresentava una minaccia in grado di sconvolgere gli equilibri di un sistema economico ancora in larghissima misura legato a un'agricoltura di sussistenza. La chiamata alle armi rappresentò un elemento di destrutturazione delle comunità rurali, lasciate alla cura quasi esclusiva di donne e anziani. Con occhi diversi guardava all'avvicinarsi della guerra la giovane intellighenzia lituana che da un breve conflitto sperava di poter strappare per i territori della cosiddetta Lituania etnica uno statuto autonomo entro i confini dell'Impero russo. L'obiettivo non rappresentava una novità. Già a cavallo tra la fine del XIX e l'inizio del XX secolo, l'idea di un territorio autonomo era balenata nei programmi dei moderni partiti politici lituani. Nel 1905, l'idea era stata ribadita nelle risoluzioni finali della Grande Dieta (Didysis seimas), la prima grande kermesse dell'intellighenzia lituana svoltasi a Vilnius nel dicembre del 1905 [Motieka 1996].

La formulazione di richieste politiche non era stata un evento subitaneo, ma l'approdo di un percorso durato vari decenni. Nella prima metà del XIX secolo il revival lituano era stato l'oggetto di un interesse di carattere elitario. Sebbene proprio elementi della classe colta, specialmente appartenenti al clero cattolico, fossero stati i protagonisti della relativa

\footnotetext{
I lituani presenti nell'Impero zarista si trovavano nella quasi totalità raccolti nei governatorati di Vilnius, Kaunas e Suwałki. Sulla base dei dati del censimento del 1897, la popolazione di lingua lituana costituival'1,8\% della popolazione totale nel governatorato di Vilnius, il 9,2\% nel governatorato di Suwałki e l’11,4\% nel governatorato di Kaunas. La popolazione di lingua lituana dominava nelle aree rurali dei governatorati di Kaunas e Suwałki (rispettivamente il 71,6\% nel governatorato di Kaunas e il 58,5\% del totale), mentre rappresentava solo il 19,8\% della popolazione nel governatorato di Vilnius dove a dominare era la popolazione bielorussa (62,9\%). Si consideri, tuttavia, che ancora alla vigilia della guerra il bilinguismo e il trilinguismo risultavano estremamente diffusi specialmente nel governatorato di Vilnius, dove la coscienza nazionale risultava estremamente debole e non necessariamente legata all'identità linguistica.
} 
avanzata dell'alfabetizzazione nelle campagne fin dalla metà del XIX secolo, il movimento visse il suo punto di svolta nella seconda metà del secolo. Negli anni successivi all'insurrezione polacca del 1863-64, esso trovò nella stampa periodica uno dei suoi principali luoghi di discussione e crescita. Com'è noto, la stampa svolge un ruolo di fondamentale importanza per la diffusione del senso di appartenenza nazionale [Anderson 1991] e dei vari miti attorno ai quali il discorso nazionale viene strutturato [Smith 2009]. Nel caso lituano, la ridefinizione dell'identità e dell'alterità era avvenuta sottolineando l'esistenza di un territorio etnicamente unitario [Sahlins 1996, 266] e corrispondente alle terre occupate dal Granducato di Lituania di epoca medievale, di fatto rendendo la Polonia e i polacchi - storicamente e territorialmente legati al Granducato - i principali esponenti dell'alterità. A causa della mancanza di un proprio libero sistema educativo [Gellner 1997], delle limitazioni imposte delle autorità dell'Impero alla sfera pubblica e della conseguente impossibilità per i lituani di monumentalizzare la propria memoria nello spazio urbano o di diffonderla attraverso pubbliche manifestazioni, la stampa rimase il principale luogo di elaborazione del discorso nazionale fino allo scoppio della Prima guerra mondiale [Safronovas 2011a]. Iniziato il conflitto, allorché l'introduzione della censura di guerra diminuì nuovamente lo spazio di manovra dell'intellighenzia lituana, i significati filtrati attraverso la stampa rappresentarono un primo essenziale strumento con cui i lettori cercarono di dotare di senso avvenimenti la cui portata non aveva pari nella memoria comunicativa [Assmann 1997]2.

\footnotetext{
Nel corso degli ultimi anni, gli studi sulla memoria storica lituana si sono sviluppati in due direzioni. Da un lato, gli studi si sono concentrati sulle principali figure del medioevo lituano [Mačiulis 2005; Nikžentaitis 2002]. Dall'altro notevole è stata l'attenzione prestata al rapporto tra la memoria storica e le aree multiculturali (Vilnius, Klaipèda, Prussia orientale, etc.) [Bumblauskas 2009; Motuzienè, Safronovas, Strakauskaitė 2011; Nikžentaitis 2011, 2013; Safronovas 2011b, 2012]. Solo quantitativamente minore si è rivelato l'interesse rivolto al processo di memorializzazione di particolari eventi storici quali le insurrezioni del 1863 [Staliūnas 2008] e le medievali battaglie di Pilènai [Baronas, Mačiulis 2010] e Grünwald [Mačiulis, Petrauskas, Staliūnas 2012].
} 
Nonostante il processo politico che a partire dalla seconda metà del 1914 portò alla nascita dello Stato nazionale Lituano sia stato l'oggetto di vari studi ${ }^{3}$, le strategie discorsive utilizzate dagli intellettuali lituani per l'interpretazione del conflitto e l'informazione dell'opinione pubblica tra l'estate del 1914 e l'inizio dell'occupazione tedesca nell'estate dell'anno successivo non hanno incontrato l'attenzione degli studiosi. Il presente intervento intende colmare almeno parzialmente questo vuoto. Mi focalizzerò, in particolare, su come le scelte di campo fatte dall'élite politica lituana all'inizio del conflitto non solo siano state presentate come una tappa necessaria nel fluire teleologico della storia nazionale, ma come esse abbiano attualizzato alcuni luoghi della master narrative storica lituana che in quegli anni l'intellighenzia stava elaborando. Attraverso l'ausilio della stampa periodica del tempo cercherò di illustrare come le argomentazioni utilizzate per dare un senso al conflitto durante i suoi primi mesi divennero il paradigma utilizzato per interpretare la successiva occupazione tedesca dei territori zaristi riorganizzati nel cosiddetto Ober Ost.

\section{II presente del passato: la Guerra come mito}

Nell'ultimo quarto del XVIII secolo, la Repubblica nobiliare polaccolituana (Rzeczpospolita) perse definitivamente la sua pluricentenaria indipendenza venendo smembrata e incorporata dagli Imperi asburgico, prussiano e russo. Le spartizioni del 1775, 1793 e 1795 non posero semplicemente fine all'esistenza di un corpo politico unitario, ma divennero il punto di partenza di una lotta tra i vari gruppi che ne contendevano la tradizione politica e culturale che si protrasse per tutto il XIX secolo. Da un lato, i diversi contesti politici in cui le varie parti

Vedi: Gimžauskas 2003; Laurinavičius 1992; Lopata 1996; Łossowski 1985; Senn 1959. 
della Repubblica furono inserite ne condizionarono i rispettivi sviluppi socioeconomici e politici. Dall'altro, il venir meno dei vincoli cetuali provocò il progressivo sfaldamento del tradizionale principio di cittadinanza fondato sull'esistenza di una nazione politica nobiliare nel rispetto delle appartenenze regionali e, in certa misura, linguistiche [Kiaupa et al. 2000]. Nei territori toccati all'Impero russo, i cambiamenti sociali ed economici furono fortemente influenzati dalle crescenti tensioni tra gli eredi della classe politica della Rzeczpospolita e l'apparato imperiale. $\mathrm{Fu}$ in particolar modo dopo le insurrezioni del 1863-64 che le misure politiche adottate dalle autorità imperiali non mirarono più solo al contenimento dell'elemento polacco (e specialmente del ceto possidente), ma misero in pratica azioni variamente indirizzate all'acculturazione dei gruppi etnici che proprio in quel periodo stavano formando una propria distinta identità socioculturale [Staliūnas 2007; Weeks 1996, 2001]. Un simile processo interessava il movimento nazionale lituano che in quel torno d'anni stava rielaborando la propria identità su base etnoculturale. Nonostante nella prima parte del XIX secolo il movimento nazionale lituano avesse già vissuto un primo sviluppo tra ristrette élite colte, le misure adottate dai centri del potere zarista dopo le insurrezioni del 1863-64 (in primis, l'ukaz sull'utilizzo dell'alfabeto latino nella resa grafica del lituano) ne interruppero la diffusione. Tuttavia, fu proprio durante il periodo di validità del divieto di stampa del lituano in caratteri latini (1864-1904) che il movimento nazionale lituano ampliò la propria base sociale [Hroch 1985, 86-97] entrando, a cavallo tra XIX e XX secolo, nella sua fase di elaborazione politica. Tra l'ultimo decennio

\footnotetext{
Nonostante la proibizione di utilizzo dell'alfabeto latino per il lituano scritto nei territori dell'Impero russo, il suo utilizzo non cessò. I principali luoghi di produzione della stampa lituana in caratteri latini divennero le cittadine lituane della Prussia orientale [Merkys 1994]. Una volta stampati, libri e riviste venivano introdotti e diffusi clandestinamente nei territori lituani dell'Impero russo grazie all'attività dei knygnešiai (portalibri). Secondo i calcoli effettuati da D. Kaunas [1996, 668] durante il periodo di validità del divieto sono state edite in Prussia orientale ben 2.687 pubblicazioni in lingua lituana e caratteri latini, delle quali 2.000 destinate ai lituani dell'Impero russo.
} 
del XIX secolo e lo scoppio del Primo conflitto mondiale il movimento vide differenziarsi al proprio interno i maggiori raggruppamenti che sarebbero diventati la spina dorsale del sistema politico dello Stato lituano interbellico. Accanto alla sinistra, che già prima della guerra si diede organizzazione partitica (Partito Socialdemocratico Lituano e Partito Democratico di Lituania), crebbero in quegli anni attorno a riviste quali «Draugija», «Viltis» e «Vairas» la destra nazionalista di Antanas Smetona e il nucleo del futuro Partito Cristiano Democratico [Gaidys 1991; Miknys 1991, 1995; Vilčinskas 1985].

A partire dagli anni ' 80 del XIX secolo la stampa clandestina in caratteri latini era diventata il principale luogo di elaborazione delle basi culturali e ideologiche del movimento nazionale lituano [Krapauskas 2000]. Dopo la relativa liberalizzazione delle strutture politiche dell'Impero zarista di inizio '900 e la legalizzazione della stampa in caratteri latini, i periodici divennero, assieme alle rappresentazioni teatrali inscenizzate con crescente frequenza specialmente in ambito rurale, uno dei principali strumenti per la nazionalizzazione delle masse contadine [Maknys 1972]. In un periodo in cui la conflittualità tra i movimenti nazionali lituano e polacco stava raggiungendo picchi mai toccati in precedenza [Merkys 2006], i giornali e le inscenizzazioni teatrali divennero mezzi per la diffusione dei principali luoghi della memoria nazionale [Nora 1989]. A differenza dei polacchi, che potevano ritrovare nel passato recente delle insurrezioni del 1794 e del 1863-64 dei memorabilia capaci di ravvivare il senso di appartenenza dei vari strati sociali a una stessa comunità nazionale, i lituani ricercarono le radici storiche della propria identità in un'epoca molto precedente.

La coscienza storica del movimento nazionale lituano si sviluppò infatti attorno a un centrale lieu de mémoire - la fioritura medievale del Granducato di Lituania dei secoli XIII-XIV. Quello medievale aveva rappresentato, secondo i membri del movimento, l'unico vero periodo di gloria dello Stato lituano prima che l'unione di Lublino (1569) e i rafforzati legami con la Polonia ne decretassero la progressiva "polonizzazione" 
linguistica, culturale e politica, fino al definitivo crollo della Repubblica di fine 700. Tra i luoghi della memoria variamente connessi con tale età dell'oro, l'attenzione dell'intellighenzia lituana si soffermò con particolare insistenza sulla Battaglia di Grünwald (1410). La battaglia, vinta da un esercito di coalizione russo-lituano-polacco, aveva rappresentato l'apice dell'opposizione del Granducato alle incursioni dei Cavalieri teutonici sulle coste del Baltico [Mačiulis et al. 2012, 12-21]. Nel discorso lituano, tuttavia, la memoria della battaglia sottolineava altri aspetti. Seguendo le logiche interne alla master narrative nazionale, la battaglia veniva letta non tanto come l'evento che grazie al quale le terre di Polonia e Lituania si erano definitivamente affrancate dalle incursioni teutoniche, quanto piuttosto come la massima vittoria riportata delle truppe lituane di Vytautas, il granduca fautore di una rinnovata autonomia del Granducato dalla Polonia di Ladislao Jagellone e in cui il nazionalismo lituano aveva riconosciuto il campione dell'antipolonismo [Nikžentaitis 2002]. La vittoria riportata contro i Cavalieri teutonici veniva pertanto trasfigurata nella celebrazione della lituanità (etnica) e del massimo condottiero lituano di sempre. Non minore, d'altro canto, poteva dirsi l'importanza della battaglia per la memoria storica polacca. Nel discorso polacco di inizio Novecento, tuttavia, la battaglia di Grünwald rimaneva uno dei momenti di gloria dello Stato lituano-polacco. Ad essere il vero perno di quell'avvenimento e il suo principale protagonista era stato non un condottiero, ma lo Stato che l'unione personale tra i ducati lituani e la corona di Polonia (Unione di Kreva, 1385) aveva creato e di cui l'esercito era espressione.

Sebbene per buona parte del XIX secolo la costante minacciosa presenza della questione polacca aveva rappresentato uno dei fondamentali problemi cui i centri di potere dell'Impero zarista avevano spesso risposto con la forza, lo scoppio del conflitto mondiale rese necessario un cambiamento di tattica. Nell'agosto 1914 la necessità di assicurarsi la fedeltà dei polacchi spinse il Capo di stato maggiore dell'esercito russo a emettere un comunicato con cui i polacchi venivano incitati a unirsi 
alla lotta contro la Germania. In cambio, veniva promessa ai polacchi l'unione di tutte le loro terre sotto lo scettro dello zar con la garanzia di una (non meglio specificata) autonomia e libertà in campo culturale e religioso. Non è certo un caso che in tale comunicato gli alti ranghi dell'esercito russo si rivolgessero ai polacchi non in termini di generica fedeltà politica, ma richiamandosi alla certezza che "[...] ancora non s'è arrugginita la spada che sconfisse il nemico presso Grünwald $»^{5}$. Sebbene il riferimento non facesse mistero della reinterpretazione in chiave panslavista della battaglia del $1410^{\circ}$, la memoria di Grünwald rappresentava uno strumento di mobilitazione cui il centro imperiale difficilmente avrebbe potuto rinunciare.

Se il riferimento alla battaglia di Grünwald iniziò con rinnovato vigore a diffondersi nel discorso pubblico polacco, non differente si rivelò la situazione in quello lituano [Mačiulis, Staliūnas, Petrauskas 2012, 67-70]. Guidati dalla convinzione di poter finalmente ottenere l'autonomia per i territori lituani dell'Impero zarista e, in caso di sconfitta della Germania, di potervi annettere le terre etnicamente lituane della Prussia orientale, già nell'agosto 1914 i lituani espressero la propria fedeltà all'Impero con la cosiddetta "Dichiarazione ambrata" (Gintariné deklaracija). Significativamente, anche in essa non mancarono né i riferimenti alla battaglia di Grünwald, né all'unione di lituani e «slavi» contro i comuni nemici «teutonici» [Basanavičius, Malinauskas 1914].

I riferimenti a Grünwald e all'alleanza con gli "slavi” presenti nella Dichiarazione erano senza dubbio più il risultato del calcolo politico che non l'espressione di una radicata convinzione [Mačiulis et al. 2012, 70].

5 Jo Šviesybès vyriausiojo komandieriaus atsišaukimas i lenkus, «Rygos garsas», 6 (19) agosto 1914, n. 62.

Secondo l'interpretazione data dai panslavisti, la Russia (il battaglione di Smolensk) avrebbe giocato per la vittoria di Grünwald un ruolo centrale. Quella di Grünwald, secondo la lettura panslavista, era stata solamente la prima difesa in ordine di tempo contro un pericolo immanente - quel Germanesimo che dopo il 1870-71 aveva cominciato a minacciare l'Europa e da cui ancora a inizio XX secolo la Russia si ergeva a difensore nei confronti del mondo slavo. 
Non sarebbe tuttavia corretto ridurre l'apporto del mito in un momento tanto importante a una semplice citazione a fini adulatori in una dichiarazione ufficiale. Tra l'estate del 1914 e la primavera del 1915, infatti, i riferimenti alla battaglia di Grünwald apparvero sulla stampa periodica lituana con una certa frequenza. A livello di comunicazione sociale, l'utilizzo diffuso di uno dei luoghi centrali della master narrative nazionale lituana ebbe due distinte funzioni. Da un lato, un così celebre luogo dell'epopea nazionale rappresentava un importante strumento per la mobilitazione della popolazione. Attraverso il raffronto del conflitto mondiale con la battaglia di Grünwald, gli intellettuali lituani cercarono di inserire negli schemi di una normalità scandita dalle tappe della teleologia nazionale un evento le cui atrocità non avevano precedenti, se non con la sola parziale eccezione della guerra russo-giapponese [Gibelli 2009]. La guerra come tale fornì l'occasione per porre l'accento sull'originaria natura pacifica dei lituani cui storicamente avevano posto fine solo le necessità di autodifesa [Dovydaitis 1914a]. In consonanza con la master narrative elaborata nei decenni precedenti e di fatto condivisa senza rilevanti distinzioni da tutte le parti della società civile definitasi etnicamente lituana ${ }^{7}$, i ducati lituani medievali - della cui tradizione il movimento nazionale si considerava l'esclusivo erede legittimo - vennero presentati come il modello del massimo equilibrio raggiunto tra le due entità metastoriche di oriente e occidente incarnate nella contemporaneità dalle due formazioni impegnate nel conflitto: i "teutoni"/"tedeschi” e i "russi”.

\footnotetext{
7 Le file del movimento nazionale erano caratterizzate dalla polarizzazione tra la maggioranza, sostenitrice di un'idea di Lituania come unità etnicamente coesa e storicamente assorbita dalla Polonia dopo l'Unione di Lublino (1569), e un'esigua minoranza, che pur riconoscendo la Lituania come un conglomerato etnico guardava ai secoli di vita comune del Granducato di Lituania con la Polonia come alla premessa per un nuovo concetto di cittadinanza su base territoriale. La corrente di maggioranza poteva dirsi la pressoché totale detentrice del monopolio della carta stampata e della comunicazione sociale nel suo complesso. Sul piano delle interpretazioni del passato storico, i sottogruppi di tale maggioranza - destra clericale, nazionalista e sinistra non presentavano differenze di sostanza.
} 
D'altro canto, se al massimo della sua potenza il Granducato di Lituania seppe resistere alle spinte di polacchi e russi grazie alla propria particolare capacità di sintesi politico-culturale, i polacchi vennero nuovamente additati come i "colpevoli" del progressivo indebolimento di dette qualità lituane, poiché dalla salita di Ladislao Jagellone al trono di Polonia diedero inizio alla "polonizzazione" dello Stato [Dovydaitis 1914b]. Nel discorso lituano di inizio conflitto, l'accento antipolacco mirava, di fatto, a mettere in guardia dalle manovre dei vicini, possibilmente volte a impedire la concessione dell'autonomia alla Lituania etnica a guerra terminata [Kraujalis 1914b]. La sottolineatura della posizione mediana dei lituani tra oriente e occidente trasformava, invece, la linea del fronte nel campo a-storico del loro stesso secolare martirio. "Le nazioni slave e i tedeschi - scrisse nel 1914 l'intellettuale cattolico Pranas Dovydaitis sono eterni e inconciliabili nemici. L'una non può guardare ai successi economici e di altro carattere dell'altra senza provare sentimenti d'invidia e vendetta. La crescita dell'una è legata all'annientamento dell'altra» [Dovydaitis 1914a]. In una simile situazione, l'attualizzazione di alcuni momenti centrali dell'epopea del medioevo lituano rendevano onore alle sofferenze della popolazione quali parti di una rinnovata battaglia di Grünwald. Quest'ultima, piuttosto, veniva presentata con accenti nuovi come un'opera lasciata incompiuta da secoli e che i lituani, contrastando i nuovi crociati tedeschi, avevano l'onorevole compito di portare a termine nel secolo XX ${ }^{8}$. Nel complesso, la nuova chiamata alle armi articolatasi sulla stampa periodica lituana si mosse tra due poli complementari. La salvaguardia dei valori della nazione etnica contadina si rifletteva nel ruralismo di sapore patriarcale degli inviti a difendere la terra dei padri (come entità economica e morale) [Bakšys 1914] dall'avanzata dei deturpatori della civiltà. La partecipazione attiva dei lituani all'incipiente Guerra mondiale diventava una nuova tappa nella costruzione dell'equilibrio europeo, della definitiva separazione tra "russi" e "teutoni" di cui

Pabaigtinas darbas, «Viltis», 24 luglio (6 agosto) 1914. 
un'entità autonoma lituana sarebbe stata coronamento e garanzia. La nazione stessa era poi chiamata a farsi protagonista in prima persona della nuova pagina dell'epopea nazionale non solo attraverso il ricordo del proprio passato, ma per mezzo della memorializzazione del proprio presente, annotando memorie e pensieri da inviare alle redazioni dei giornali e raccogliere in appositi archivi [Bugailiškis 1914; Landsbergis 1914; Papentis 1915].

\section{L’attualizzazione del mito: i lituani, i tedeschi e la Guerra}

Il processo di attivazione e attualizzazione della memoria storica dei lituani, d'altro canto, fu accompagnato da un accurato aggiornamento dell'immagine dell'alterità tedesca. Nei fatti, tale aggiornamento consistette nel tentativo di razionalizzare il legame esistente tra i Cavalieri teutonici e l'Impero tedesco entro l'ampio recinto della categoria astorica di germanesimo quale sinonimo di violenza, terrore e rapina. Ne uscì un quadro in cui la classe politica tedesca risultava essere, per matrice genetica, l'origine stessa di una tirannia di cui erano state vittime prima i tedeschi, poi l'Europa'.

All'inizio del conflitto, nei resoconti giornalistici il processo di unificazione tedesco fu illustrato come la prima tappa del nuovo percorso che aveva portato al disvelamento della violenza prussiano-teutonica. In consonanza con un habitus iscritto nel loro plurisecolare patrimonio politico, gli Hohenzollern «[...] ottennero la guida della Germania non perché la loro cultura o la loro civiltà superasse in qualche misura quella delle altre Terre Germaniche, ma solo in virtù della loro forza fisica, della loro spada, in una parola sola, delle loro guerresche attitudini» [Noreika 1914]. La forza bruta iscritta nel patrimonio politico dei prussiani si era dunque riversata prima di tutto sui loro stessi connazionali. Tuttavia,

9 Kaip atsirado Vokietija, «Šaltinis», 8 settembre (26 agosto) 1914. 
a trasformarla in un pericolo reale per tutta l'Europa non era bastata la conquista (e non l'unificazione) dell'Impero tedesco da parte dei prussiani. La nascita del pangermanesimo, l'ideologia che aveva fatto dei tedeschi un nuovo "popolo eletto» [Vailokaitis 1915b], appariva piuttosto come l'escrescenza della grandiosa crescita industriale del paese [Abraitis 1914]. In presenza di una secolare cultura politica improntata alla violenza, la nascita di una grande potenza industriale, che del genio prussiano appariva come l'indubbio frutto, non poteva, secondo gli osservatori lituani, che condurre a una rinnovata corsa agli armamenti e a un nuovo ciclo espansivo all'insegna dell'empietà [Markauskas 1914b]. Seppur legata a un passato che ne tratteneva già in nuce tutti i caratteri salienti, la Germania appariva, tuttavia, come lo specchio di una nuova era e, ancor più, di una nuova Europa tesa tra il pauroso clamore della tecnologia e l'invidiato sviluppo dello stato-nazione. Grazie alla tecnologia, le dimensioni della distruzione causata dalla macchina bellica si erano dilatate in una misura precedentemente inimmaginabile. Se la tecnologia, tuttavia, risultava caratterizzata da una fondamentale neutralità morale che la rendeva uno strumento che l'uomo poteva utilizzare per il proprio sviluppo socioeconomico, ad essere incriminata era l'intenzione di chi se facesse uso a fini definibili come immorali. La guerra meccanizzata che l'Impero tedesco aveva scatenato incarnava pertanto l'escrescenza della malvagità teutonica indirizzata alla narcisistica realizzazione di un sé collettivo ${ }^{10}$ a detrimento delle nazioni vicine. Se la guerra mondiale era dunque nuova in quanto a mezzi impiegati, ma non in relazione alla volontà di chi la guerra l'aveva causata, mutati erano, invece, i soggetti che ai nuovi Cavalieri teutonici si potevano e volevano opporre. Per la prima volta nell'era moderna, infatti, ad affrontarsi erano non semplicemente gli stati, ma le nazioni. Da un lato, nonostante alcune nazioni (prima tra tutte quella tedesca) avessero già ben dimostrato nel corso del secolo precedente di essere coese, la

10 Kur artimo meilè? «Aušra», 29 ottobre 1914. 
guerra era parsa un particolare stimolo alla sintesi per tutte le nazioni europee [Markauskas 1914a]. In svariate occasioni i colonnisti fecero riferimento all'inusuale spinta all'unità che lo scoppio del conflitto esercitò nei confronti delle diverse anime politiche degli stati coinvolti. All'inizio del conflitto, le frizioni ideologiche precedentemente presenti in Russia, Francia, Inghilterra e Germania, riferiva un commentatore, erano sparite con fulminea rapidità tanto da far sembrare il conflitto mondiale un'autentica guerra delle nazioni («tautu karas») [Vailokaitis 1914]. Dall'altro, l'accento posto sul carattere nazionale del conflitto confermava implicitamente le aspettative riposte in una veloce vittoria russa. Come i tentativi di coordinare le varie correnti politiche lituane nell'autunno 1914 dimostrano, l'intellighenzia lituana - specialmente conservatori e clericali - vedeva nell'unità sovrapartitica il prerequisito di qualsiasi possibile successo politico dei lituani. Accanto a tali auspici di unità politica, che conseguirono un parziale successo solo dopo l'invasione tedesca dell'estate 1915, la novità di una guerra tra nazioni si presentava come l'inevitabile epilogo delle dispute iniziate in tutta Europa (e fuori di essa) nel corso del XIX secolo. Una guerra di nazioni rappresentava - anche concettualmente - l'ultimo atto della vita di quegli stessi stati multinazionali che della guerra erano i protagonisti. Al di là della sua matrice tecnologica, pertanto, la guerra era nuova in quanto vero epilogo dell'ancien régime e prologo di un nuovo ordine mondiale dominato da soggettività nazionali. L'idea fu ben espressa nel febbraio 1915 di Juozas Vailokaitis. In un intervento pubblicato settimanale Šaltinis, Vailokaitis inserì il conflitto - compendiando una teoria per nulla originale - nella morfologia di sviluppo, fioritura e decadenza della storia universale. La storia universale, affermava Vailokaitis, dimostra come anche gli imperi che godettero della massima fama e del massimo rispetto tra i propri cittadini inevitabilmente perirono per mano dei loro alleati o di particolari settori della loro stessa società. Il conflitto segnava la raggiunta vecchiaia di alcuni stati e la prima giovinezza di quelle forze che nel corso del XIX secolo erano cresciute e che, durante 
il conflitto, avrebbero offerto nuova linfa alla lotta controDichiarazione ambrata la barbarie tedesca [Vailokaitis 1915a]. Nella fattispecie, con la "Dichiarazione ambrata" i lituani avevano offerto la loro lealtà non come l'atto dovuto dei sudditi nei confronti del loro zar, ma come il contributo volontario dei membri di un'individualità nazionale interessata e attivamente impegnata alla difesa dei propri interessi comunitari [Paparnis 1914]. Gli interessi nazionali, appunto. Per quanto non esplicitamente dichiarato, nel concetto di interesse nazionale presente sulla stampa riecheggiò chiaramente una critica del rapporto tra centro imperiale, nazioni presenti nel territorio dell'Impero e i loro diritti positivi. Non può certo meravigliare che le critiche fossero rivolte alle potenze nemiche di Germania e Austria-Ungheria, mentre la Russia rimaneva al di fuori della discussione grazie alle misure di autocensura adottate dalle testate stesse. La critica passava, invece, attraverso la citazione di casi in cui il rapporto tra centro e periferia pareva essere esemplare: "[Il colonialismo inglese] non ha introdotto limitazioni alla religione, alla lingua e nemmeno alla possibilità di governare il proprio paese, ma ha solamente preteso che venissero acquistati i prodotti inglesi e che all'Inghilterra venisse venduto quanto eventualmente prodotto» [Kraujalis 1914a]. Se, tra le righe, è possibile leggere una critica alle opposte tendenze tenute dalle autorità zariste nei confronti dei lituani dopo la rivolta del 1863-64, essa non toccava l'Impero come forma di stato, ma i rapporti tra il centro e le sue componenti nazionali intese come soggettività politico-culturali. Essa cioè evidenziava il nocciolo di un nuovo possibile patto politico tra l'apparato burocratico imperiale e le nazioni quali depositarie del potere di rappresentanza degli individui, sottolineando ancora una volta come la concessione dell'autonomia alle nazioni dell'impero costituisse l'unica via per un rinnovato patto tra lo stato e i suoi cittadini. Implicitamente, tale riequilibrio tracciava il nuovo limite tra potenze europee: da un lato, gli imperi multinazionali in cui la forza dello Stato si accorda alla cooperazione economica tra i suoi cittadini e al rispetto dei diritti positivi delle nazioni; dall'altro, le potenze accentratrici - l'Impero tedesco in 
primis - in cui la potenza economica rappresenta il risultato di un'omologazione forzata e una spinta all'espansionismo imperialistico.

Il conflitto - cui i lituani partecipavano come nazione di matrice contadina - veniva, infine, letto anche come base per un profondo mutamento dei rapporti sociali esterni e interni allo Stato. Se la guerra era stata il risultato dell'azione di «[...] industriali, possidenti terrieri e, in generale, classi dominanti [...] totalmente incuranti dei bisogni delle classi inferiori» [Markauskas 1914c] e delle masse di contadini tedeschi senza terra [Untulis 1915], la sconfitta degli imperi centrali avrebbe portato al mutamento di quella struttura sociale che la Germania aveva ereditato dalla Prussia e di cui il paese era la massima espressione storica. Al suo posto si sarebbe potuto affermate un nuovo assetto di potere comune sia ai paesi sconfitti che all'Impero zarista. Simili considerazioni si basavano sulla convinzione che il fondamentale apporto alla vittoria della Russia zarista sarebbe arrivato proprio dalle giovani nazioni presenti nell'impero e il cui riconoscimento ufficiale, come il caso delle promesse fatte ai polacchi rendevano oramai evidente, non sarebbe più stato rinviabile [Markauskas 1914d].

\section{Dal mito alla realtà: l'esperienza dell'occupazione tedesca}

Fino alla nuova avanzata tedesca sul fronte orientale nella primavera-estate del 1915, il mito e la sua attualizzazione rappresentarono un utile strumento per rendere relativamente familiare alle

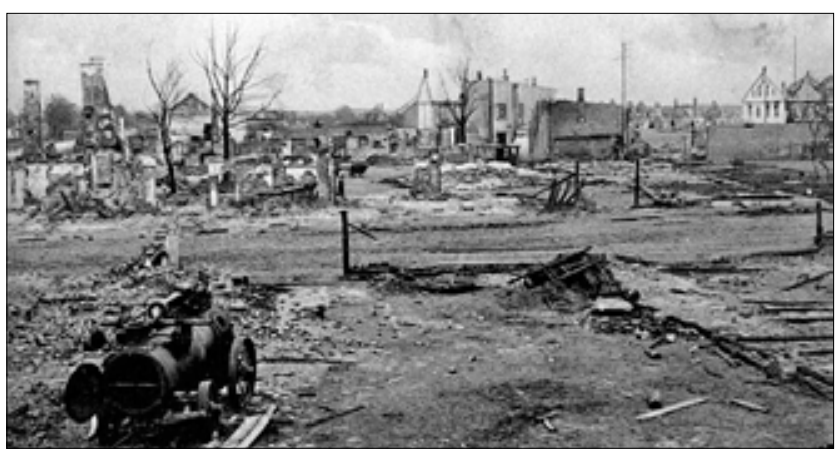

Cittadina lituana in fiamme durante l'avanzata tedesca, 1915 (fonte: Lietuvos dailes muziejus: http://www.epaveldas.lt/vbspi/ biRecord.do?biRecordId=9267) 
masse contadine un evento senza precedenti. Ma se attraverso l'utilizzo del mito e della sua attualizzazione la classe intellettuale lituana aveva cercato di mobilitare la popolazione in vista di una veloce sconfitta della Germania, il mutamento della situazione e l'inizio dell'occupazione tedesca scombinò irrimediabilmente gli scenari prospettati. Per circa 500.000 abitanti di quelli che erano stati i Territori del Nord-ovest dell'Impero russo, l'estate del 1915 coincise con l'emigrazione verso le regioni centrali della Russia ${ }^{\prime \prime}$. Per questi ampi strati di popolazione - tra cui circa 250.000 lituani - si trattò dell'inizio di un periodo di "esilio" che rese tuttavia possibile lo sviluppo di un reticolo di strutture educative, assistenziali e burocratiche di dimensioni mai viste prima. Seppur tra restrizioni notevolmente maggiori, il periodo dell'occupazione tedesca del cosiddetto Ober Ost rappresentò un'analoga occasione per la popolazione rimasta in loco. Va però notato che mentre per i lituani dislocati in Russia il periodo dell'occupazione tedesca fu principalmente un'occasione di formazione civica e politica, per chi rimase nell'Ober Ost il quotidiano rapporto con l'amministrazione militare e civile tedesca contribuì a rafforzare la percezione di sé in contrapposizione all'alterità degli occupanti. Su questo ultimo punto concentrerò la mia attenzione. Se nell'estate del 1914 la guerra aveva rappresentato un evento concreto e palpabile solo per le popolazioni residenti a ridosso della linea del fronte, l'inizio dell'occupazione e l'insediamento delle nuove strutture amministrative tedesche tra la tarda primavera e l'autunno del 1915 resero concretamente chiara a tutti la natura degli avvenimenti che stavano interessando il resto dell'Europa e del mondo. Nonostante, come abbiamo visto, le ragioni della guerra venissero principalmente rinvenute nell'imperialismo tedesco e nel suo sviluppo tecnologico-industriale, la

\footnotetext{
11 Di tutti gli abitanti della regione di occupazione tedesca (Ober Ost) trasferitisi nelle regioni centrali della Russia, solo 350.000 circa poterono far ritorno nel neonato Stato indipendente lituano tra il 1918 e il 1923. Molti rifugiati videro il loro ritorno reso difficile o addirittura impossibilitato dalle pratiche introdotte dalle autorità lituane e miranti a impedire il ritorno di elementi variamente inaffidabili o in grado di mutare pericolosamente gli equilibri etnici dello Stato: Balkelis 2004.
} 
fascinazione per uno Stato che nel giro alcuni decenni era riuscito a crearsi e a salire ai vertici economici e politici d'Europa poteva essere difficilmente nascosta. La "modernità" tedesca appariva ancor più sensazionale se confrontata al grado di arretratezza della Russia zarista e ai suoi crescenti problemi sociali. L'avanzata dell'esercito tedesco, tuttavia, fu decisiva per cancellare ogni residua fede nei buoni frutti della "civiltà tedesca” e ravvivare l'immagine del nemico come l'epigono dei Cavalieri teutonici e dei loro rudi modi. Il sacerdote Kazimieras Pakalniškis [1940, 105] espresse nel suo diario il contrasto tra le residue speranze e l'esperienza diretta dell'occupazione nei termini di un amaro disincanto in contrasto con quanto di buono sul conto dei tedeschi era potuto filtrare in precedenza:

Prima della guerra avevo letto sulla stampa lituana e polacca del grande timor di Dio dei tedeschi, di tutte le loro associazioni e organizzazioni deputate all'innalzamento dello spirito e delle condizioni materiali della gente, delle loro vivaci e solenni assemblee annuali. Ero convinto che quei cattolici tedeschi dovessero essere, nella loro vita di ogni giorno, delle persone eccezionali, un vero esempio per i cattolici di tutte le nazioni. È passato più di un anno da quando vivo nel "territorio di occupazione tedesca” (Im besetzen Gebiete). Mi è capitato di vivere per più tempo tra i soldati tedeschi. Tra di loro v'erano sia cattolici che protestanti, ma io non ho notato tra le loro condotte di vita differenza alcuna. [...] Quando c'era da derubare la nostra gente, da violentare donne e ragazze, i soldati tedeschi cattolici partecipavano e in nulla si differenziavano dai loro colleghi protestanti. I cattolici bavaresi erano i più violenti e crudeli di tutti i soldati, gli stessi tedeschi li chiamavano i "cosacchi di Germania”.

Nella diaristica e nella memorialistica delle classi popolari (e, a dire il vero, non solo delle classi popolari) l'accostamento tra nuovi occupanti e Cavalieri teutonici venne declinato in termini ancora meno mediati. Ne sono un buon esempio le memorie di Ona Tuomènaitė che ancora nel 1937 argomentava l'occupazione tedesca nei termini di una vendetta per la sconfitta subita a Grünwald [Ruseckas 1939, 285]. Durante gli anni dell'occupazione, i soldati dell'esercito tedesco apparvero simili a 
"belve" (žverys) che «depredarono la nostra terra e [...] nel peggiore dei modi si comportarono con le nostre ragazze» [Čilvinaitè 1937, 200], introdussero «nei confronti degli abitanti l'ordine della crudeltà tedesca e i modi del governo teutonico [...]» basato sulla «[...] rapina e l'uccisione di pacifici paesani [...]» [Urbelis 1926, 127].

Ma cosa aveva potuto causare un così forte senso di rigetto per l'alterità tedesca e una così stretta associazione con la violenza dei Cavalieri teutonici? La questione è già stata trattata altrove [Gimžauskas 2010; Griffante 2014; Liulevičius 2000]. Mi limiterò a indicarne qui alcune delle principali ragioni. In primo luogo, nell'Ober Ost le forze di occupazione avevano visto un territorio dal cui sfruttamento avrebbe tratto beneficio tutta l'economia di guerra tedesca. Le requisizioni del bestiame e dei generi alimentari introdotte fin dal principio dell'occupazione - e che assunsero ben presto un carattere massiccio - avevano esattamente il doppio obiettivo di soddisfare, almeno in parte, i bisogni dell'esercito e della popolazione tedesca [Mašiotaitė-Urbšienè 1939]. Accanto alle requisizioni vere e proprie e alla miriade di balzelli introdotti col passare del tempo [Gimžauskas 2010], i soldati di stanza sul territorio non esitarono fin da subito a effettuare ulteriori sottrazioni di beni d'ogni genere a propria totale discrezione e spesso non lesinando atti di gratuita violenza fisica e psicologica. I prodotti alimentari non rappresentarono l'esclusivo oggetto dell'interesse tedesco. Durante gli anni dell'occupazione, le foreste divennero l'oggetto di uno sfruttamento sistematico senza precedenti che defraudò parte del territorio di una delle sue principali fonti economiche. Tuttavia, ad essere oggetto e strumento dello sfruttamento furono soprattutto gli abitanti locali utilizzati vieppiù come forza lavoro gratuita. La tendenza all'utilizzo forzato dei civili nelle attività economiche si era evidenziata fin dai primissimi giorni dell'occupazione [Žadeikis 1921, 65]. Corvée a cadenze più o meno regolari nei manieri fatti propri dalle forze militari tedesche divennero una consuetudine cui gli abitanti furono obbligati, loro malgrado, ad abituarsi [Westerhoff 2012]. Il volume della popolazione impegnata per periodi consecutivi in 
servizi di lavoro - di fatto, veri e propri lavori forzati - nell'Ober Ost o in Germania aumentò considerevolmente tra il 1916 e il 1917. Obbligati a prestar servizio in campi di lavoro sguarniti di qualsiasi più elementare norma igienica per mesi o addirittura anni, ammassati in locali malsani, malnutriti e spesso malati, migliaia di abitanti dell'Ober Ost esperirono sulla propria pelle tutta la durezza del regime di occupazione [Griffante 2013; Westerhoff 2012].

L'occupazione tedesca contribuì, in secondo luogo, alla decostruzione dell'universo morale lituano. Non tenendo conto delle festività religiose che caratterizzavano il calendario di una società agraria come quella lituana, i vari servizi di lavoro obbligatorio scardinarono la distinzione tra tempo sacro e tempo profano. Similmente, lo spazio sacro si ritrovò ad essere materialmente violato dalla presenza tedesca ${ }^{12}$.

La popolazione $\mathrm{fu}$ inoltre oggetto di un controllo che superò di gran lunga le restrizioni conosciute durante il periodo zarista. Enormi limitazioni furono imposte alla libera circolazione delle persone sul territorio, vincolando i movimenti alle nuove entità amministrative (Bezirke) o al nullaosta delle autorità. In un periodo caratterizzato da requisizioni, razionamento dei generi alimentari e da una progressiva mancanza di beni di prima necessità, le limitazioni alla mobilità resero gli approvvigionamenti ancor più difficoltosi. Nel corso del 1917, le difficoltà di approvigionamento diminuirono la razione media degli abitanti di Vilnius a sole 805 calorie giornaliere e a un aumento della mortalità dal 2,2\%

12 Particolarmente impressionante per la popolazione locale era il disinvolto utilizzo dello spazio sacro a fini a dir poco profani. Come ricorda Jurgis Lapinskas [1939, 204], durante una visita al paese di Plutiškès, a sud est di Kaunas, la visione della chiesa riisulta sconcertante: "Premetti la maniglia. La porta si aprì. Entrai all’interno e vidi con mio grande stupore che la nostra chiesetta era stata trasformata in una scuderia. Puzzava di urina di cavallo e le pareti erano state tutte rosicchiate dai cavalli. Anche se il locale era stato ripulito dallo sterco, l'odore di sterco era molto intenso». Situazioni smili erano molto comuni in tutto il territorio di occupazione. 
del 1915 al 8,13\% del novembre $1917^{13}$. Nel complesso, tale situazione trasformò gli anni dell'occupazione tedesca dei territori dell'Ober Ost in «[...] una dei peggiori e più tristi periodi della nostra storia» durante il quale «[...] i lituani hanno compreso di che stoffa siano fatti quei loro vicini d'occidente che si consideravano i portatori di cultura per l'oriente» [Mažuika 1935, 297].

\section{Conclusioni}

Le brevi considerazioni presentate in queste pagine sono probabilmente sufficienti per dimostrare come nel corso del Primo conflitto mondiale l'immagine dei tedeschi come dei nuovi Cavalieri teutonici sia stata l'oggetto di una singolare eterogenesi dei fini. Se nell'estate e nell'autunno del 1914 l'immagine era stata utilizzata con il malcelato obiettivo di mobilitare la popolazione nella convinzione di una veloce vittoria russa, in seguito all'avanzata dell'esercito tedesco e alla successiva occupazione essa divenne un paradigma in grado di spiegare e render ragione della durezza delle misure adottate dagli occupanti.

Va tuttavia detto che per quanto l'esperienza del periodo 1915-1918 e lo stereotipo violento del tedesco rimase chiaramente impressa nella memoria degli abitanti che subirono sulla propria pelle le conseguenze dell'occupazione, essa divenne nel corso del dopoguerra la vittima di una progressiva cancellazione dalla sfera pubblica che continua fino ai nostri giorni. Le ragioni possono essere ricondotte a tre ordini di fattori. In primo luogo, l'esperienza dell'occupazione tedesca riguardò solo una parte dei futuri cittadini della Repubblica di Lituania nata nel 1918 e fu pertanto patrimonio della memoria comunicativa di una sola parte della nuova nazione politica. Per le decine di migliaia di persone rimpatriate

\footnotetext{
13 Biblioteca dell'Accademia Lituana delle Scienze, fondo 255-991, 1. Comunicazione alla Lietuvos Taryba del novembre 1917.
} 
dalla Russia dopo la fine del conflitto, la memoria della guerra mondiale rimase associata all'evacuazione nelle regioni interne dell'Impero zarista e non a sofferenze materiali paragonabili a quelle esperite da chi rimase nelle proprie abitazioni. Fino allo scoppio rivoluzionario, le comunità emigrate in Russia poterono condurre una vita nei limiti del possibile normale e con una libertà d'azione - ad esempio in campo educativo e culturale - superiore a quella avuta prima dell'inizio del conflitto.

In secondo luogo, la disputa lituano-polacca relativa alla regione di Vilnius e la sua presenza nell'agenda internazionale per tutti gli anni '20 e '30 rese il ruolo dell'occupazione tedesca meno centrale per la memoria collettiva rispetto a quanto non lo fossero la Polonia (la Rzeczpospolita) e i polacchi. Proprio in questi ultimi la master narrative storica lituana aveva individuato i principali nemici della nazione, storicamente impegnati nell'opera di "polonizzazione" dei lituani fin dal XIV secolo.

In terzo luogo, nel corso del dopoguerra la memoria della dolorosa occupazione tedesca e l'immagine dei tedeschi come nuovi Cavalieri teutonici avrebbe potuto oscurare o mutare il significato del principale luogo della memoria dello Stato lituano indipendente: la battaglia di Grünwald. Ad essa, nella sua accezione di vittoria dei lituani (e non di un esercito plurinazionale) sui tedeschi e al loro principale condottiero, Vytautas, fu dedicato il massimo risal to nello spazio pubblico lituano per tutto il periodo interbellico. Se le impellenze dell'agenda politica dello Stato nazione lituano spiegano le ragioni dell'oblio di questa pagina di storia del Baltico orientale, esse non ne possono più rappresentare una giustificazione. Questo contributo vuole rappresentare la ricostruzione di un piccolo tassello di una problematica complessa che solo ora, a cent'anni di distanza gli storici professionisti hanno iniziato a studiare. 


\section{Fonti}

Abraitis J. 1914, Karui prasidèjus, «Lietuvos ūkininkas», 25 luglio (7 agosto).

Bakšys J. 1914, Lietuvi, mylèk savo žemelę, "Aušra», 1 ottobre.

Basanavičius J., Malinauskas D. 1914, Lietuviu deklaracija, «Rygos garsas», 27 agosto (9 settembre).

Bugailiškis P. 1914, Rinkime karo meto medžiagą!, "Lietuvos ūkininkas», 21 settembre (1 ottobre).

Dovydaitis P. 1914a, Lietuva ir karas, "Viltis», 13 (26) ottobre.

Dovydaitis P. 1914b, Tarp kūjo ir priekalo, «Viltis», 3 (16) settembre.

Čilvinaitè M. 1937, Didžiojo karo vargai Šaukènu apylinkëje. (Iš Šaukènu kanauninko kūnijo Jono Staškevičiaus pasakojimu). «Karo archyvas», 8: 198-207.

Kraujalis P. 1914a, Mažosios tautos, "Aušra», 14 settembre.

Kraujalis P. 1914b, Lietuviai budèkime!, "Aušra», 17 settembre.

Landsbergis G. 1914, Didžiojo karo istorijai, «Lietuvos žinios», 10 (23) dicembre.

Lapinskas J. 1939, Mano atsiminimai iš okupacijos laiku, «Karo archyvas», 11: 201-213.

Markauskas M. 1914a, Didysis Europos karas, LŪ, 14 (27) agosto.

Markauskas M. 1914b, Prūsu Lietuva, «Lietuvos ūkininkas», 28 agosto (10 settembre).

Markauskas M. 1914c, Vokietija, «Lietuvos ūkininkas», 11 (24) settembre.

Markauskas M. 1914d, Karas ir mažesniosios tautos, «Lietuvos ūkininkas», 6 (19) novembre.

Mašiotaitė-Urbšienė M. 1939, Vokiečiu okupacijos ūkis Lietuvoje, Kaunas.

Mažuika J. 1935, Didžiojo karo atsiminimai, «Karo archivas», 6: 292-297.

Noreika L. 1914, Iš ko kilo karas, "Vairas», 30 agosto.

Pakalniškis K. 1940, Rusų-vokiečiu karo užrašai, «Karo archyvas», 12: 95-147.

Paparnis [?] 1914, Lietuvos reikalai ir reikalavimai, «Viltis», 1 (14) novembre.

Papentis [?] 1915, Rašykime karo istorija, «Saltinis», 30 maggio (12 giugno).

Ruseckas P. 1939, Lietuva Didžiajame kare, Vilnius: Vilniaus žodis.

Untulis M. 1915, 1915, «Lietuvos ūkininkas», 8 (21) gennaio.

Urbelis A. 1926, Vokiečiu okupacijos laikai, «Karo archyvas», 3: 112-127.

Vailokaitis P. 1914, Šio karo ypatybès, «Šaltinis», 30 (17) novembre.

Vailokaitis P. 1915a, Didysis karas. Jo kilmè ir prasmè, «Šaltinis», vasario 8 (sausio 26).

Vailokaitis P. 1915b, Išrinktoji tauta, «Šaltinis», 14 (27) maggio.

Žadeikis P. 1921, Didžiojo karo užrašai. I dalis, Klaipèda: Lituania. 


\section{Bibliografia}

Anderson B. 1991, Imagined Communities: Reflections on the Origins and Spread of Nationalism, London: Verso (I ed. 1983).

Assmann J. 1997, Das kulturelle Gedächtnis. Schrift, Erinnerung und politische Identität in frühen Hochkulturen, München: Beck.

Balkelis T. 2004, In Search of a Native Realm: The Return of World War One Refugees to Lithuania, 1922-24, in Baron N., Gatrell P. (ed.), Homelands: war, population and statehood in Eastern Europe and Russia, 1918-1924, London: Anthem Press, 74-97.

Baronas D., Mačiulis D. 2010, Pilenai ir Margiris: istorija ir leganda, Vilnius: Sapnų sala.

Bumblauskas A. (ed.) 2009, Naujasis Vilniaus perskaitymas: didieji Lietuvos istoriniai pasakojimai ir daugiakultūris miesto paveladas, Vilnius: Vilniaus universiteto leidykla.

Gaidys A. 1991, Lietuvių krikšćioniu demokratu partijos kūrimosi aplinkybès (1905-1907 m.), in Lietuvos atgimimo istorijos studijos 3: Lietuvos valstybès idëja (XIX a.-XX a. pradžia), Vilnius: Zaltvykslè, 139-172.

Gellner E. 1996, Nazioni e nazionalismo, Roma: Editori riuniti.

Gibelli A. 2009, L'officina della guerra: la grande guerra e le trasformazioni del mondo mentale, Torino: Bollati Boringhieri.

Gimžauskas E. 2003, Baltarusiu veiksnys formuojantis Lietuvos valstybei 1915-1923 m., Vilnius: LII leidykla.

Gimžauskas E. 2010, Vokieči karinès okupacijos poveikis Lietuvos visuomenei ir besiformuojančiam valstybingumui 1915-1919 m., "Karo archyvas», 25: 98 -123.

Griffante A. 2013, A New Master and a New Serfdom: Understanding the Compulsory Labour Experience of Lithuanians During the German Occupation, 1915-1918, "Res Balticae», 12: 91-105.

Griffante A. 2014, We and Homeland: German Occupation, Lithuanian Discourse, and War Experience in Ober Ost, in J. Bürgschwentner, M. Egger, G. Barth-Scalmani, Other fronts, other wars? First World War Studies on the Eve of the Centennial, LeidenNew York: Brill, 237-255.

Hroch M. 1985, Social Preconditions of National Revival in Europe: A Comparative Analysis of the Social Composition of Patriotic groups among the smaller European $\mathrm{Na-}$ tions, Cambridge: Cambridge University Press.

Kaunas D. 1996, Mažosios Lietuvos knyga. Lietuviškos knygos raida, 1547-1940 m., Vilinius: Baltos lankos.

Kiaupa Z., Kiaupienė J., Kuncevičius A. 2000, Lietuvos istorija iki 1795 metų, Vilnius: A. Varnas.

Krapauskas V. 2000, Nationalism and Historiography: the case of Nineteenth-Century Lithuanian Historicism, Boulder (Colo.): Columbia University Press. 
Laurinavičius Č. 1992, Lietuvos-Sovietu Rusijos Taikos sutartis: 1920 m. liepos 12 d. sutarties problema, Vilnius: Valstybinis leidybos centras.

Liulevičius V. G. 2000, War Land on the Eastern Front: Culture, National Identity, and German Occupation in World War I, Cambridge: Cambridge University Press.

Lopata R. 1996, Lietuvos valstybingumo raida 1914-1918 metais, Vilnius: Mintis.

Łossowski P. 1985, Po tej i tamtej stronie Niemna: stosunki polsko-litewskie, 1883-1939, Warszawa: Czytelnik.

Mačiulis D., Petrauskas R., Staliūnas D. 2012, Kas laimëjo Žalgirio mūššr? Istorinio paveldo dalybos Vidurio ir Rytu Europoje, Vilnius: Mintis.

Mačiulis D. 2005, Valstybès kultūros politika Lietuvoje 1927-1940 metais, Vilnius: Lietuvos Istorijos instituto leidykla.

Maknys, V. 1972, Lietuvių teatro raidos bruožai, Vilnius, Mintis.

Merkys V. 1994, Knygnešiu laikai, 1864-1904, Vilnius: Valstybinis leidybos centras.

Merkys V. 2006, Tautiniai santykiai Vilniaus vyskupijoje 1798-1918, Vilnius: Versus Aureus.

Miknys R. 1991, Vilniaus autonomistai ir ju 1904-1905 m. Lietuvos politines autonomijos projektai, in Lietuvos atgimimo istorijos studijos 3: Lietuvos valstybès ideja (XIX a.-XX a. prad žia), Vilnius: Žaltvykslè, 173-198.

Miknys R. 1995, Lietuvos demokratu partija 1902-1915 metais, Vilnius: A. Varnas.

Motieka E. 1996, Didysis Vilniaus Seimas, Vilnius: Saulabrolis.

Motuzienė L., Safronovas V., Strakauskaitė N. (ed.) 2011, Daugiareikšmés tapatybès tarpuerdvèse: Rytu Prūsijos atvejis XIX-XX amžiais, Klaipėda: Klaipėdos universiteto leidykla.

Nikžentaitis A. 2002, Vytauto ir Jogailos juaizdis Lietuvos ir Lenkijos visuomenese, Vilnius: Aidai.

Nikžentaitis A. (ed.) 2011, Nuo Basanavičiaus, Vytauto Didžiojo iki Molotovo ir Ribbentropo: Atminties ir atminimo kultūru transformacijos XX-XXI amžiuje, Vilnius: Lietuvos Istorijos instituto leidykla.

Nikžentaitis A. (ed.) 2013, Atminties daugiasluoksniškumas: miestas, valstybè, regionas, Vilnius: Lietuvos Istorijos instituto leidykla.

Nora P. 1991, Between Memory and History: Les Lieux de Mémoire, "Representations», 26: 7-24.

Safronovas V. 2011a, Kultūrine atmintis ar atminimo kultūra? Kultūrinès atminties teorijos taikymo moderniuju laiku tyrimams problemos, in Nikžentaitis A. (ed.) Nuo Basanavičiaus, Vytauto Didžiojo iki Molotovo ir Ribbentropo: Atminties ir atminimo kultūru transformacijos XX-XXI amžiuje, Vilnius: Lietuvos Istorijos instituto leidykla, 39-64. 
Safronovas V. 2011b, Praeitis kaip konfliktu šaltinis: tapatybès ideologijų konkurencija XX amžiaus Klaipedoje, Vilnius: Lietuvos Istorijos instituto leidykla.

Safronovas V. (ed.) 2012, Erdviu pasisavinimas Rytu Prūsijoje XX amžiuje, Klaipèda: Klaipedos universiteto leidykla.

Sahlins P. 1996, Frontières et identités nationales, Paris: Berlin.

Senn E. A. 1959, The Emergence of Modern Lithuania, New York: Columbia University Press.

Smith A. D. 2009, Ethno-Symbolism and Nationalism: A cultural approach, LondonNewYork: Routledge.

Staliūnas D. 2004, Europos idèja lietuviškame XIX-XX a. sandūros diskurse, in Staliūnas D. (ed.), Europos ideja Lietuvoje: istorija ir dabartis, Vilnius: LII leidykla, 2004, 105114.

Staliūnas D. 2007, Making Russians: Meaning and Practice of Russification in Lithuania and Belarus after 1863, Amsterdam-New York: Rodopi.

Staliūnas D. 2008, Savas ar svetimas paveldas? 1863-1864 m. sukilimas kaip lietuvin atminties vieta, Vilnius: Mintis.

Vilčinskas J. 1985, Lietuvos socialdemokratija kovoje dèl krašto nepriklausomybès: istoriné apžvalga, London: S. Mockus ir S. Bosikis.

Weeks T. R. 1996, Nation and State in Late Imperial Russia: Nationalism and Russification on the Western Frontier 1863-1914, De Kalb: Nothern Illinois University Press.

Weeks T. R. 2001, Russification and the Lithuanians, «Slavic Review», 60 (1): 96-114.

Westerhoff C. 2012, Zwangsarbeit im Ersten Weltkrieg: deutsche Arbeitskräftepolitik im besetzten Polen und Litauen, 1914-1918, Padeborn: F. Schöningh. 